\title{
Positron Emission Mammography (PEM) in the diagnosis of breast cancer: A systematic review and economic evaluation
}

\author{
Khosro Keshavarz ${ }^{1}$, Mojtaba Jafari ${ }^{2}$, Farhad Lotfi ${ }^{1}$, Peivand Bastani ${ }^{1}$, Mahmood Salesi ${ }^{3}$, Farshid Gheisari ${ }^{4,5}$, \\ Mohsen Rezaei Hemami*6 (10)
}

Received: 3 Sep 2019

Published: 18 Aug 2020

\section{Abstract}

Background: Positron Emission Mammography (PEM) is an imaging technique which is increasing focuses on imaging the chest instead of imaging the whole body. The aim of this study was to conduct a systematic review of the clinical efficacy and costeffectiveness of PEM technology, as compared with PET, as a diagnostic method used for breast cancer patients.

Methods: The present study was a Health Technology Assessment (HTA), which was conducted via a systematic review of clinical efficacy and cost-effectiveness of the methods based on domestic evidence. To evaluate the efficacy of the PEM diagnostic method, as compared with PET, we used efficacy indices, including Sensitivity, Specificity, Accuracy, PPV, and NPV. The required data were collected through a meta-analysis of studies published in electronic databases from 1990 to 2016. In addition, direct costs in both methods were estimated and finally, a cost-effectiveness analysis was performed using the results of the study. Also, a one-way sensitivity analysis was performed to examine the effects of parameters' uncertainty in the model. In this study, we used STATA software to integrate the results of studies with similar parameters.

Results: A total of 722 cases $(\mathrm{N})$ were obtained from the five final studies. The results of the meta-analysis performed on the collected data showed that the two methods were identical in terms of the Specificity and PPV parameters. However, as to Sensitivity, NPV, and Accuracy parameters, the PEM method was superior to the PET for diagnosis of primary breast cancer. The total cost of using PEM and PET was $\$ 1737385.7$ and $\$ 1940903.5$, respectively, and the cost of a one-time scan (cost per unit) using PEM and PET devices was $\$ 86.82$ and $\$ 157.63$, respectively. As compared with the PET method, the use of the PEM diagnostic method for diagnosis of breast cancer was cost-effective in terms of all the five studied parameters (it was definitely cost-effective for four parameters and was also considered as cost-effective for another index, since ICER was below the threshold).

Conclusion: The results showed that the use of PEM technology for the diagnosis of primary breast cancer is more cost-effective than PET technology; thus, due to the wide range of PET technology in different fields, it is recommended that this method should be used in other areas of priority.

Keywords: Meta-analysis, Breast cancer, PEM, PET, HTA

Conflicts of Interest: None declared

Funding: Iran National Institute of Health Research, Tehran, Iran

\section{*This work has been published under CC BY-NC-SA 1.0 license.}

Copyright $\odot$ Iran University of Medical Sciences

Cite this article as: Keshavarz Kh, Jafari M, Lotfi F, Bastani P, Salesi M, Gheisari F, Rezaei Hemami M. Positron Emission Mammography (PEM) in the diagnosis of breast cancer: A systematic review and economic evaluation. Med J Islam Repub Iran. 2020 (18 Aug);34:100. https://doi.org/10.47176/mjiri.34.100

Corresponding author: Dr Mohsen Rezaei Hemami, mohsen.rezaeihemami@abdn.ac.uk

1. Health Human Resources Research Center, School of Management and Medical Informatics, Shiraz University of Medical Sciences, Shiraz, Iran

2. Student Research Committee, Shiraz University of Medical Sciences, Shiraz, Iran

3. Chemical Injuries Research Center, Systems Biology and Poisonings Institute, Baqiyatallah University of Medical Sciences, Tehran, Iran

4. Faculty of Medicine, Shiraz University of Medical Sciences, Shiraz, Iran

5. Ionizing and Non-lonizing Radiation Protection Research Center, School of Paramedical

Sciences, Shiraz University of Medical Sciences, Shiraz, Iran

6. University of Exeter, PenTAG Health Technology Assessment, UK $\uparrow$ What is "already known" in this topic:

PEM focuses on breast imaging rather than imaging the entire body. The results of recent studies suggest that PEM may be a useful diagnostic tool and, therefore, requires further evaluation.

$\rightarrow$ What this article adds:

This study is the first systematic review of efficacy and costeffectiveness that aimed to evaluate the PEM diagnostic strategy, as compared with PET, in the diagnosis of breast cancer in Iran. The results showed that the use of PEM for the diagnosis of primary breast cancer is more cost-effective than PET technology. The policymakers, health managers, researchers, etc. can use the result of this study. 


\section{Introduction}

Cancer is defined as a condition where body cells are threatened due to uneven cell growth (1); it is associated with uncontrolled growth and spread of the abnormal cells, which, if not controlled, will lead to death (2). The cancer incidence and mortality rates in 182 countries are published by the International Agency for Research. Taking into consideration the most common cancers in general, about 12.7 million new cases of cancer and 7.6 million deaths from cancer occurred in 2008. Breast cancer, as the second most common cancer in the world, next to the lung cancer, accounted for 1.38 million cases of cancers $(10.9 \%$ of all cancers $)(3,4)$.

Breast cancer is the most common cancer among women in all countries of the world. The incidence of this cancer is increasing, especially in developing countries, as it has risen 3 to 4 times over the last few decades (5-8). The prevalence of breast cancer among women in Iran over a period of 30 years was $17.1 \%$ on average (9), and it was reported as the fifth cause of death among women (10).

Due to its relatively high prevalence, the economic burden of breast cancer in most countries is high; for instance, its economic burden in the United States was estimated to be $\$ 947,374,468$ in 2010 , of which $77 \%$ was related to the costs incurred by death from breast cancer. Hence, correct and timely diagnosis and treatment of the cancer that reduces mortality can greatly reduce the burden of the disease $(7,11)$. For example, the incidence of breast cancer in the United States has been rising over the past years, but mortality has decreased due to early diagnosis and treatment (12). The methods used for the diagnosis of breast cancer include the following: clinical examination, mammography, ultrasound, breast MRI (Magnetic Resonance Imaging), biopsy, PET (Positron emission tomography) scan, or PEM (Positron emission mammography). Mammography is the most commonly used method in Iran, but in young women ultrasound is used more (13).

PET is a new and advanced imaging technique that, using a radio tracker, detects biological processes including cellular behaviors performed for metabolism and cell proliferation. PET is increasingly used to diagnose different types of cancer, especially breast cancer, and may be used as an auxiliary or alternative imaging technique for screening and diagnosing vulnerable patients who, for different reasons, cannot tolerate MRI or other technologies (14-16). Furthermore, PET technology is a safe technique for diagnosis of primary breast cancer $(17,18)$, except for some specific patients, including pregnant women, women who breastfeed their baby, diabetic patients, as well as highly overweight people who cannot get into the devices (17, 19 and 20).

However, PEM focuses on breast imaging rather than imaging the entire body. PEM costs are lower than normal PET costs (21). PEM has a higher spatial resolution and takes less time than PET $(21,22)$. The results of recent studies suggest that PEM may be a useful diagnostic tool and, therefore, requires further evaluation (23). It also has an acceptable level of efficacy; for example, in a study by
Wendy et al. which was conducted to evaluate the diagnosis of breast cancer using PEM technology, the results showed that the sensitivity, specificity, and accuracy of PEM for the diagnosis of different types of breast cancer were $90-91 \%, 86-93 \%$, and $88-92 \%$, respectively (19).

Therefore, PEM is the best choice for screening vulnerable patients (24), as studies on this technology have shown that it is safe and without adverse side effects (2527). Nevertheless, as the use of technology incurs some costs on the health system (28) while the resources are limited, the widespread use of this technology or any other new technology in the healthcare system requires constructive interactions with the authorities and the implementation of Health Technology Assessment (HTA) studies (29-31). Therefore, the aim of this study was to compare PEM technology with PET as a breast cancer diagnostic method in terms of efficiency and costeffectiveness in Iran.

\section{Methods}

\section{Systematic Review}

\section{Data Resources and Search Strategy}

In order to evaluate the efficiency of PEM and PET methods for early diagnosis of breast cancer, we conducted a systematic review of electronic databases including PubMed, Scopus, Web of Science, and Cochrane Library and searched for the relevant studies published between 1990 and 2016.

The following combinations of the keywords were used:

\#1-(((positron Emission Mammography) OR (PEM)) AND ((breast cancer) OR (Breast Neoplasms) OR (breast carcinoma) OR (breast tumor)))

\#2-(((Positron Emission Tomography) OR (PET)) AND ((breast cancer) OR (Breast Neoplasms) OR (breast carcinoma) OR (breast tumor)))

\#3-(((Positron Emission Tomography) OR (PET)) AND ((Positron Emission Mammography) OR (PEM)))

\#4- \#1 OR \#2 OR \#3.

\section{Inclusion and Exclusion Criteria}

The inclusion criteria were the randomized clinical trials published in English that compared the clinical efficiency of PEM with PET for the diagnosis of primary breast cancer.

The exclusion criteria included studies on animals, studies without control groups, observational studies, review studies, econometric studies, studies not approved by Ethics Committee, and irrelevant articles.

\section{Study selection}

The PRISMA guideline was used to conduct our systematic review (32). First, the total number of articles that met the inclusion criteria was determined, and the results of all searches in the listed sites were transferred to the Endnote X7 software. Then, the duplicated items found in different sites were deleted. Afterward, two researchers individually reviewed the titles and abstracts of the search results and removed unrelated articles and the articles 
which did not meet the inclusion criteria. In the next step, the results obtained by the two researchers were matched, the fulltexts of the selected articles were studied, and the articles containing the required data were selected. In addition, a third reader resolved discrepancies between the two researchers (Fig. 1).

\section{Quality Assessment}

The quality of the trial studies was assessed using the Quality Assessment of Diagnostic Accuracy Studies (QUADAS-2) (33). The QUADAS-2 tool has three domains to identify the risk of bias and applicability judgments: Patient selection, Index test, and Reference standard. In the end, studies that were consistent with the listed indices had an acceptable quality (low risk in most do- mains) and had identical methodology were entered into the meta-analysis.

\section{Outcomes of the study}

In order to compare the efficiency of PEM and PET technologies, we used the following indices: Sensitivity, Specificity, Accuracy, PPV, and NPV.

\section{Data Analysis}

In this study, following the systematic review approach, first we extracted the data on the diagnostic parameters of both PEM and PET methods as well as data on sensitivity, specificity, accuracy, PPV, and NPV. Then, a metaanalysis was performed in order to integrate the results of studies with similar parameters; in this step, we used
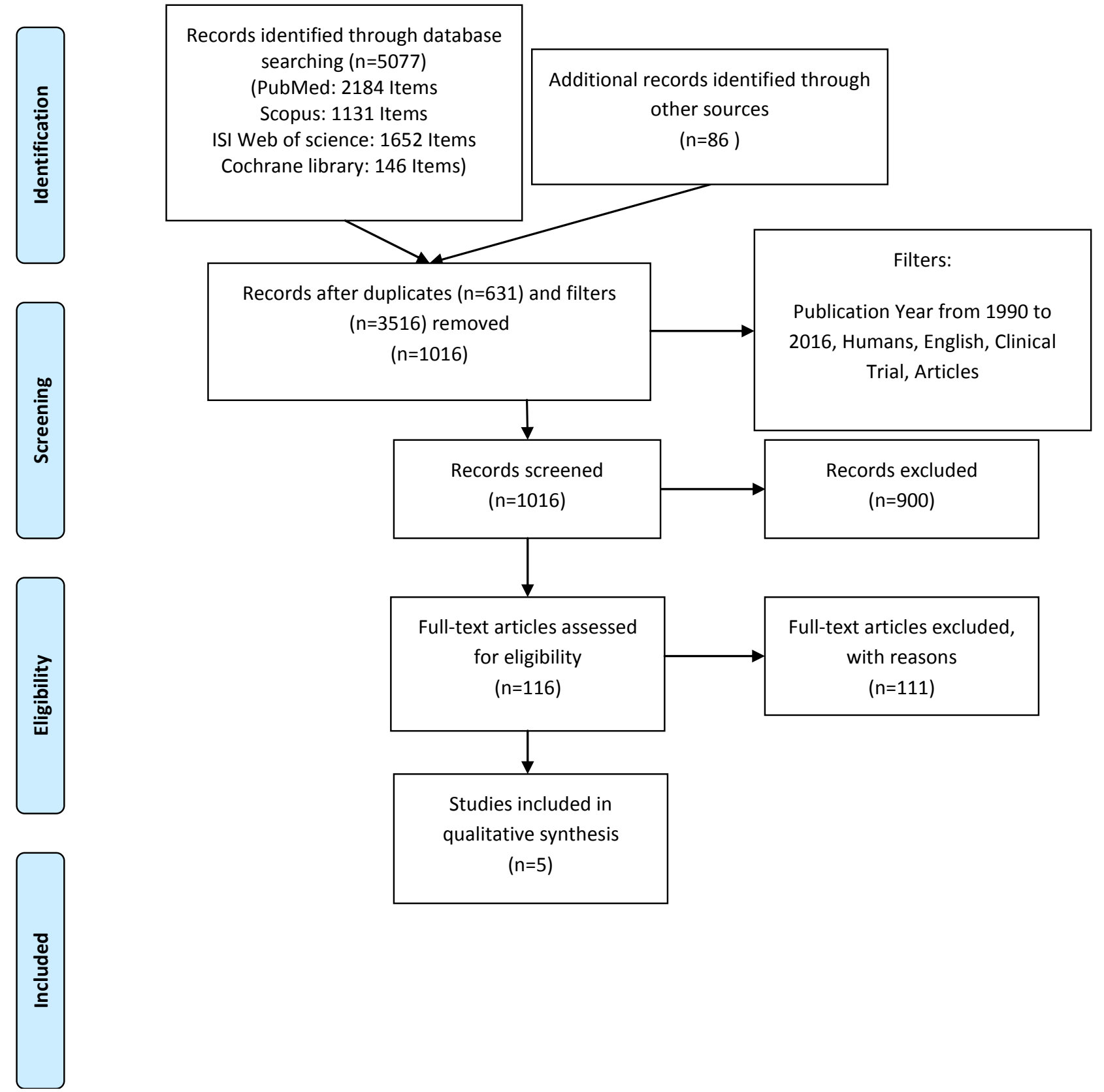

Fig. 1. Diagram of the process of selecting clinical trials which investigated the alternatives under the study 
STATA software. To test the heterogeneity, we used the Q Cochran test and a value less than 0.1 was considered statistically significant. In the case of heterogeneity or shortage of studies, the random effects method was utilized. Moreover, a forest plot was used to show the results of the meta-analysis.

\section{Cost-effectiveness analysis \\ Cost sources}

Cost items related to PEM and PET technologies include the total cost of installing and launching a PEM device versus a PET device (technology purchase costs, building costs, depreciation costs, maintenance costs, and supplies and Staff Costs). Due to the lack of PEM technology in the country, the related costs were calculated based on the prices in Iran and the exchange rates for United States Dollar to Iranian Rial in 2016, using the following items: 1 . Views of the experts about the cost of using PET and PEM technologies for the diagnosis of breast cancer, 2. A report from the company importing the PEM technology about the related costs (it was received from the HTA office of the Ministry of Health), 3. A report on PET technology assessment prepared by the HTA Office of the Ministry of Health in 2007 (34, 35).

In addition, some cost items that were not available for the year 2016 were adjusted using the following formula:

$$
\mathrm{f}=\mathrm{p}(1+\mathrm{i})^{\mathrm{n}}
$$

Where $\mathrm{n}$ is the number of years or periods, $\mathrm{I}$ is the average inflation rate from 2007 to $2015, \mathrm{P}$ is the initial value in 2007 , and $\mathrm{F}$ is the current value.

Furthermore, the number of tests was also adjusted for the variable costs.

\section{Cost items}

To calculate the cost of a one-time scan using each of the PEM and PET diagnostic devices, the following costs were taken into account:

- Costs of purchasing and installing the technology which was calculated based on the self-declaration of the providers who had the device or were going to import the device.

- Building costs which were estimated by calculating the average cost of buying a building in Tehran in 2016.

- Cost of the total space required for PEM installation and launch, including a scanning room (4 x 4) and an injection room $(2 \times 3$ and $2 \times 3)$.

- Cost of the total space required for PET installation and launch, including a scanning room (7.5 x 5.03), an injection room $(2 \times 3$ and $2 \times 3)$, and a control room (3.18 x 5.03).

- Cost of a total space of $50 \mathrm{~m}$ required for the installation of a cyclotron device.

- Cost of depreciation which was also calculated based on the national tax law of the country and the annual depreciation rate $(10 \%)$.

- Costs related to the number of scans, assuming that the PEM or PET device is active two shifts a day and 5 days a week, and each shift 15 persons for PEM and 6 persons for PET are admitted. Accordingly, an approximate number of 15600 PEM scans and 6240 PET scans were estimated for each year.

- Costs of materials and consumables (variable costs) were calculated for a total of 6240 PET scans and 15600 PEM scans. In addition, the costs of supplying materials for 21840 scans were calculated for the cyclotron. Consumables included lab supplies, chemicals, disposable injection supplies and materials, and a set of clothing for the patient.

- Staff Costs: A radiology expert is required for each of the PEM and PET devices and cyclotron in each work shift.

- Other costs include maintenance and repair, energy, marketing, training, and other possible costs.

\section{Incremental Cost-Effectiveness Ratio (ICER)}

Considering the data collected for the analysis of efficiency and cost-effectiveness, in order to make a decision, first the incremental cost-effectiveness ratio (ICER) was calculated and then it was compared with the threshold. The cost-effectiveness ratio was calculated using the following formula:

$$
I C E R=\frac{\text { Cost } t_{-} \text {PEM }- \text { Cost }_{-} \text {PET }}{\text { Effectiveness } \_ \text {PEM }- \text { Effectiven ess _PET }}
$$

\section{Uncertainty analysis}

Finally, a one-way sensitivity analysis was conducted to examine the effects of cost and effectiveness parameters uncertainty on the study results. The variables' values were increased by $20 \%$, and the tornado diagrams were drawn.

\section{Results}

The abstracts and titles of 1016 articles were reviewed and the fulltexts of 116 articles were studied (Fig. 1). Of all the reviewed articles, we finally selected five articles that had the required data and the desired indices (36-40). The total number of patients included in the selected studies was 871 patients, of whom 722 cases were evaluable for scanning. The mean age of the patients was 53.71 years. Table 1 presents the characteristics of the selected studies including the year of study, comparison arm, the number of cases evaluable for scanning, mean age of the patients, and the data on efficacy related to the diagnostic parameters of both PEM and PET methods. In addition, the quality of the studies was assessed using the QUADAS-2 tool and as the results of the study indicated, the quality of all the studies was acceptable (low Risk). (Table 1 and Fig. 2)

The results related to the efficiency of PEM methods as compared with PET

The size of the samples in all of the charts in the PEM and PET groups was equal to 722 people, and at a $\mathrm{p}<0.05$, the difference between the two groups was significant. 
Table 1. Characteristics of selected studies in the systematic review and the extracted data on efficacy related to the diagnostic parameters of both PEM and PET methods

\begin{tabular}{|c|c|c|c|c|c|c|c|c|c|c|c|}
\hline \multirow[t]{2}{*}{ Study } & \multicolumn{2}{|c|}{ Restaging tool } & \multirow{2}{*}{ 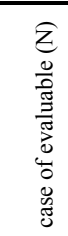 } & \multirow{2}{*}{\multicolumn{2}{|c|}{ 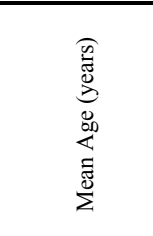 }} & \multicolumn{2}{|c|}{ Sensitivity (\%) } & \multicolumn{2}{|c|}{ Specificity (\%) } & \multicolumn{2}{|c|}{ Accuracy (\%) } \\
\hline & 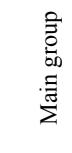 & 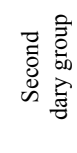 & & & & $\sum_{\text {至 }}$ & $\stackrel{-5}{\underline{1}}$ & $\sum_{\text {至 }}$ & 空 & 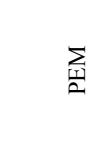 & $\stackrel{-5}{2}$ \\
\hline Yamamoto, Y. et al (2016) (36) & PEM & PET & 256 & 78.9( & & 100 & 75 & 85.08 & 85 & 86.71 & 83.59 \\
\hline Yamamoto, Y. et al (2015) (37) & PEM & PET & 108 & 42.7( & & 78.57 & 47.6 & 90.9 & 93.93 & 86.11 & 75.92 \\
\hline Eo, J. S. et al (2012) (39) & PEM & PET & 113 & $52(3$ & & 94.69 & 86.72 & & & 94.49 & 86.72 \\
\hline Schilling, K. et al (2011) (40) & PEM & PET & 67 & 59.7( & & 85 & 68 & 74.07 & 47.61 & 80.6 & 55.22 \\
\hline Kalinyak J. E. et al (2014) (38) & PEM & PET & 69 & $62(4$ & & 46.66 & 6.66 & 90.74 & 96.29 & 81.16 & 76.81 \\
\hline Kalinyak J. E. et al (2014) (38) & PEM & PET & 109 & $57(2$ & & 56.52 & 13.04 & 90.7 & 95.35 & 83.48 & 77.98 \\
\hline \multicolumn{12}{|l|}{ Table 1. Ctd } \\
\hline \multirow[t]{2}{*}{ Study } & \multicolumn{2}{|c|}{$\mathrm{TN}$} & \multicolumn{2}{|c|}{$\mathrm{FN}$} & \multicolumn{2}{|c|}{ FP } & \multicolumn{2}{|c|}{$\mathrm{TP}$} & \multirow{2}{*}{\multicolumn{3}{|c|}{$\begin{array}{c}\text { QUA } \\
\text { DAS-2 Bias }\end{array}$}} \\
\hline & $\sum_{\substack{\mid=1 \\
2}}$ & $\stackrel{-\mathfrak{n}}{\underline{2}}$ & $\sum_{\substack{\Delta \\
\Sigma}}$ & 㖉 & 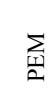 & $\stackrel{-\mathfrak{y}}{\underline{2}}$ & $\sum_{\substack{1 \\
2}}$ & $\stackrel{t}{\underline{2}}$ & & & \\
\hline Yamamoto, Y. et al (2016) (36) & 194 & 187 & 0 & 9 & 34 & 33 & 28 & 27 & & Low risk & \\
\hline Yamamoto, Y. et al (2015) (37) & 60 & 62 & 9 & 22 & 6 & 4 & 33 & 20 & & Low risk & \\
\hline Eo, J. S. et al (2012) (39) & 0 & 0 & 6 & 15 & 0 & 0 & 107 & 98 & & Low risk & \\
\hline Schilling, K. et al (2011) (40) & 20 & 20 & 6 & 8 & 7 & 22 & 34 & 17 & & Low risk & \\
\hline Kalinyak J. E. et al (2014) (38) & 49 & 52 & 8 & 14 & 5 & 2 & 7 & 1 & & Low risk & \\
\hline Kalinyak J. E. et al (2014) (38) & 78 & 82 & 10 & 20 & 8 & 4 & 13 & 3 & & Low risk & \\
\hline
\end{tabular}

A: QUADAS-2 tool

Flow and timing

Reference standard

Index test(s)

Patient selection

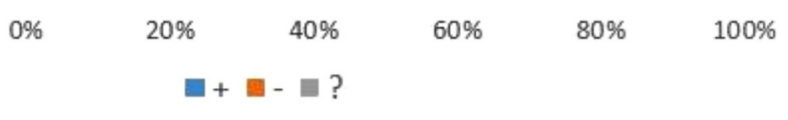

B: QUADAS-2 tool

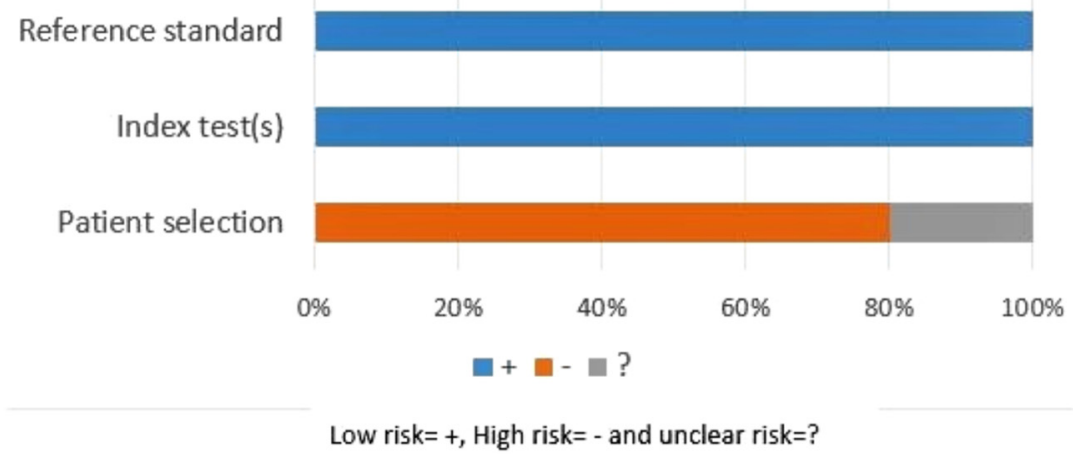

Fig. 2. The proportion of the studies with low risk, high risk, and unclear risk (A: risk of bias (\%) \& B: applicability concerns (\%))

Comparison between PEM and PET methods in terms of sensitivity

Based on Figure 3, the results of the Q Cochran test for heterogeneity measurement showed that, taking into consideration $\mathrm{I}^{2}=70.07 \%$ and $\mathrm{p}=0.004$, the studies were not homogeneous. Therefore, a random effect model was used 


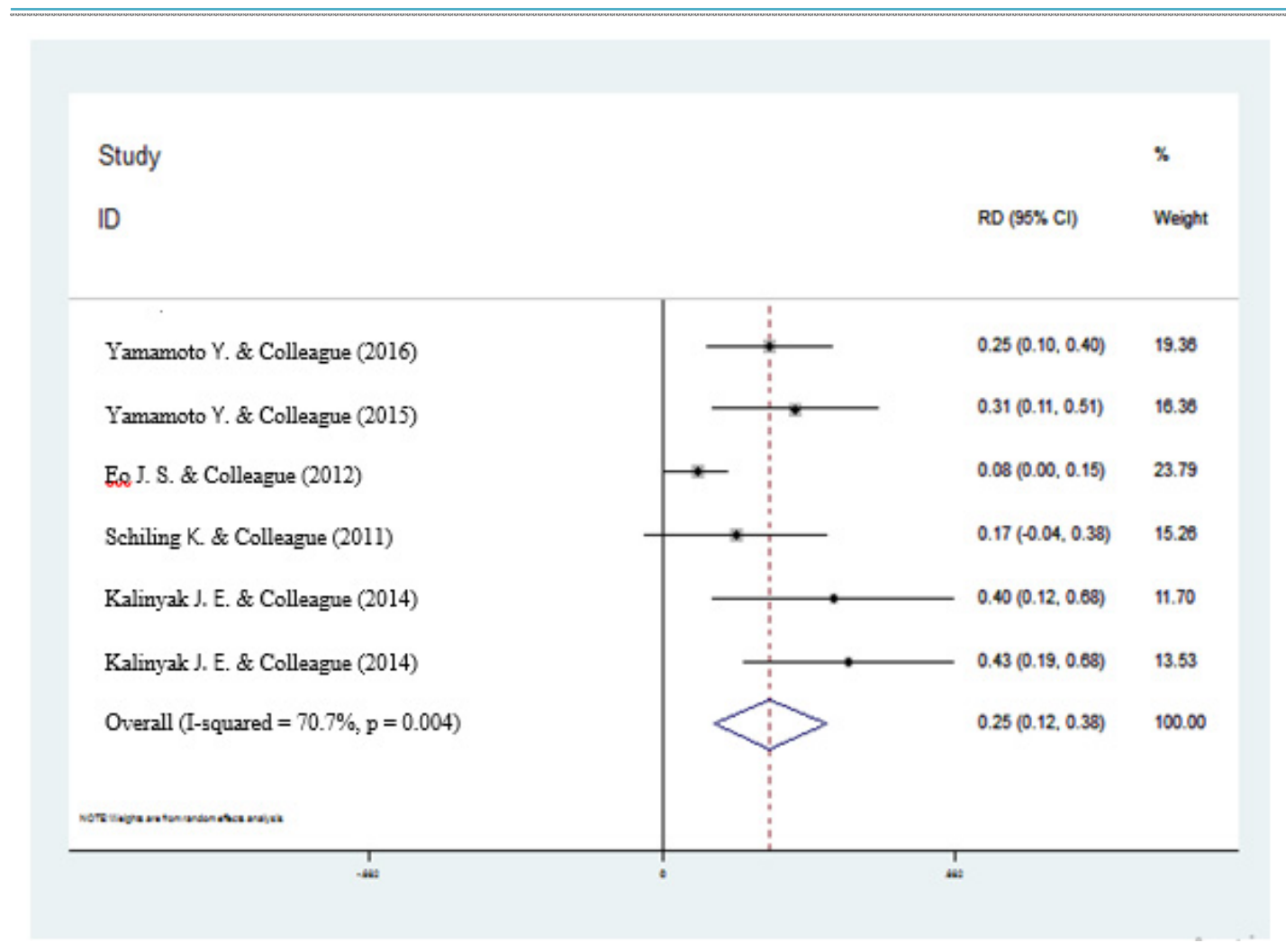

Fig. 3. Individual and Pooled Risk Difference for the Outcome of "sensitivity" in the randomized Studies Considering PEM comparing to PET

to compare the sensitivity. Overall, the difference in sensitivity between the two groups of PEM and PET was 0.25 $(0.12,0.38)$, indicating that the sensitivity of the PEM device in detecting breast cancer was $25 \%$ more than that of PET device $(\mathrm{p}=0.000)$.

Comparison between PEM and PET methods in terms of specificity

Based on Figure 4, the results of the Q Cochran test for

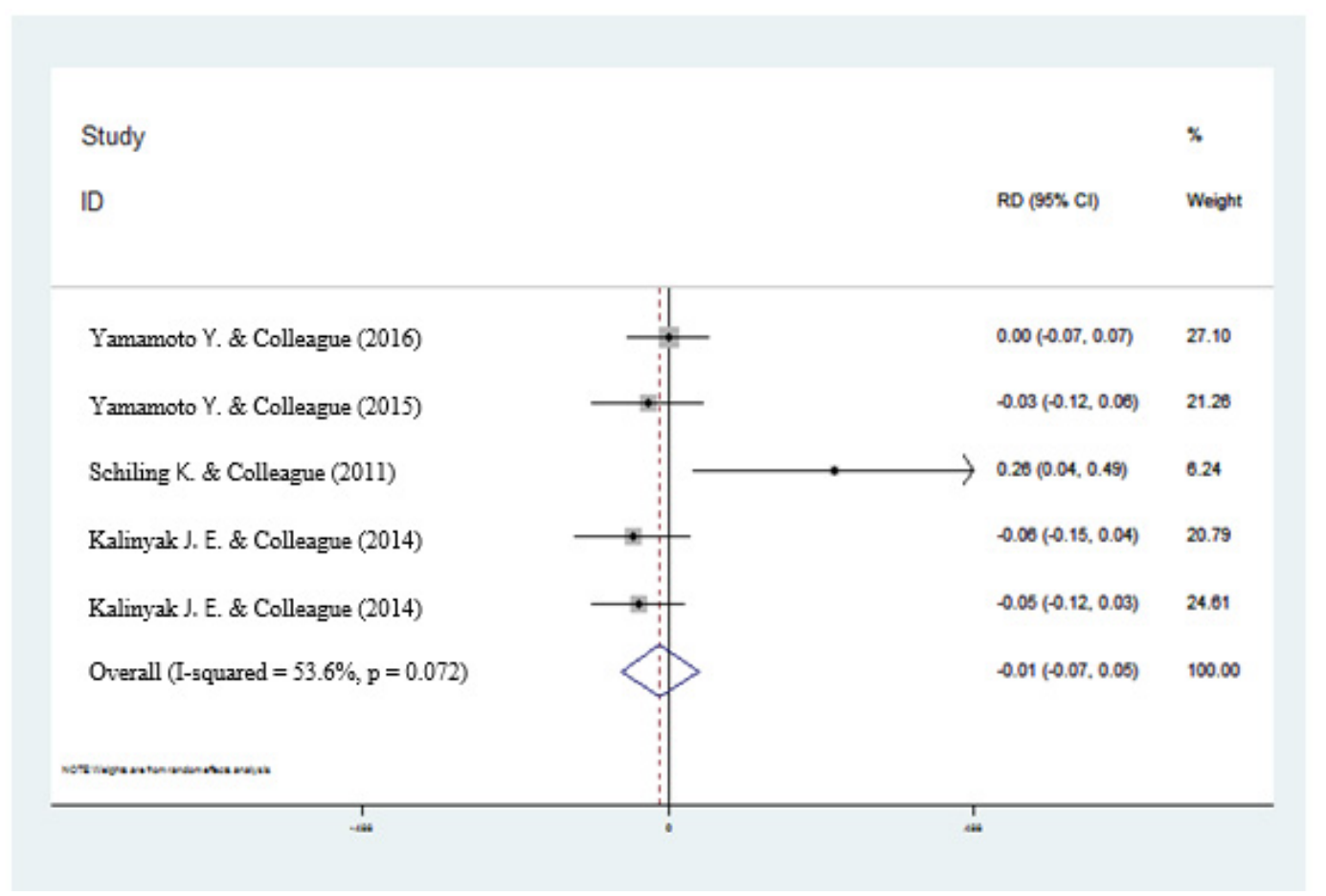

Fig. 4. Individual and Pooled Risk Difference for the Outcome of "specificity" in the randomized Studies Considering PEM comparing to PET 
heterogeneity measurement showed that, taking into consideration $\mathrm{I}^{2}=53.6 \%$ and $\mathrm{p}=0.072$, the studies were not homogeneous. Therefore, a random effect model was used to compare the specificity. Overall, the difference in specificity between the two groups of PEM and PET was -0.013 $(-0.07,0.05)$, indicating that the specificity of the PEM device in detecting breast cancer was $1.3 \%$ less than the specificity of PET device. However, PEM did not show significant differences compared to PET in terms of specificity $(\mathrm{p}=0.683)$.

\section{Comparison between PEM and PET methods in terms} of accuracy, PPV and NPV

The results of the $\mathrm{Q}$ Cochran test for heterogeneity measurement showed that, taking into consideration $\mathrm{I}^{2}=48.8 \%$ and $\mathrm{p}=0.099$, the studies were not homogeneous. Therefore, a random effect model was used to compare the accuracy. Overall, the difference in accuracy between the two groups of PEM and PET was $0.08(0.02$, $0.15)$, indicating that the accuracy of the PEM device in detecting breast cancer was $8 \%$ more than the accuracy of PET device $(\mathrm{p}=0.015)$.

Moreover, the results of PPV analysis showed that, taking into consideration $\mathrm{I}^{2}=63.2 \%$ and $\mathrm{p}=0.028$, the studies were not homogeneous. Therefore, a random effect model was used to compare PPV. Overall, the difference in PPV between the two groups of PEM and PET was 0.15 (-0.04, $0.34)$, indicating that the PPV of the PEM device in detecting breast cancer was $15 \%$ more than the PPV of PET device, but the difference between the two groups was not statistically significant $(\mathrm{p}=0.121)$.

The results of NPV analysis showed that, taking into consideration $\mathrm{I}^{2}=0.0 \%$ and $\mathrm{p}=0.449$, the studies were homogeneous. Therefore, a fixed-effect model was used to combine them. Overall, the difference in NPV between the two groups of PEM and PET was $0.07(0.03,0.11)$, indicating that the NPV of the PEM device in detecting breast cancer was 7\% more than the NPV of PET device $(p=0.00)$. The results of the meta-analysis of the studied diagnostic parameters in PEM and PET methods are summarized in Table 2.

\section{Results of analysis of the costs of PEM and PET meth-} ods

With respect to the number of PEM scans (15600) and PET scans (6240) per year and the costs of supplying radio medication for a cyclotron unit for 21840 scans per year, the costs of one-time scan (cost per unit) using PEM and PET devices without cyclotron were equal to $\$ 25.46$ and $\$ 96.26$ dollars, respectively, and the cost of a cyclotron (cost per unit) alone was $\$ 61.36$. In addition, the costs of a PEM and PET scan with cyclotron (cost per unit) were \$86.82 and \$157.63, respectively (Table 3).

\section{Results of analysis of the cost-effectiveness of PEM} and PET methods

Based on the results of Table 4, as compared with PET method, the use of PEM method reduced the cost of each scan session by $\$ 70.8$ and increased the sensitivity, accuracy, NPV, and PPV by $25 \%, 8 \%, 7 \%$, and $15 \%$ respectively, and decreased the specificity by $1.3 \%$. Therefore, since PEM is superior to PET in terms of the efficiency and is less costly, there is no need to calculate ICER for the four indices of sensitivity, accuracy, NPV and PPV. Hence, PEM is quite superior and cost-effective. The results of cost-effectiveness analysis in terms of specificity index showed that, with increasing each unit of specificity, PEM, in comparison with PET, increased the cost by

Table 2. Summary of the results of the meta-analysis of the studied diagnostic parameters in PEM and PET methods

\begin{tabular}{|c|c|c|c|c|c|c|}
\hline \multirow[t]{2}{*}{ Parameter } & \multicolumn{3}{|c|}{ Effect size } & \multicolumn{3}{|c|}{ Heterogeneity } \\
\hline & PEM- PET (Diff) & $\mathrm{p}$ & \multicolumn{2}{|r|}{$\mathrm{Chi}^{2}(\mathrm{p})$} & \multicolumn{2}{|c|}{ Method } \\
\hline \multirow{5}{*}{$\begin{array}{l}\text { Sensitivity } \\
\text { Specificity } \\
\text { PPV } \\
\text { NPV } \\
\text { Accuracy }\end{array}$} & 0.25 & $<0.001$ & \multicolumn{2}{|r|}{$17.19(.004)$} & \multicolumn{2}{|c|}{ Random effect } \\
\hline & -0.013 & 0.683 & \multicolumn{2}{|r|}{$8.61(.072)$} & \multicolumn{2}{|c|}{ Random effect } \\
\hline & 0.15 & 0.121 & \multicolumn{2}{|r|}{$10.87(.028)$} & \multicolumn{2}{|c|}{ Random effect } \\
\hline & 0.072 & $<0.001$ & \multicolumn{2}{|r|}{$3.7(.449)$} & \multicolumn{2}{|c|}{ Fixed effect } \\
\hline & 0.081 & 0.015 & \multicolumn{2}{|r|}{$7.81(.099)$} & \multicolumn{2}{|c|}{ Random effect } \\
\hline \multicolumn{2}{|l|}{ Costs } & PET & PEM & Cycltron & $\mathrm{PET}+\mathrm{Cycl}$ & $\mathrm{PEM}+\mathrm{Cycl}$ \\
\hline \multicolumn{2}{|c|}{ purchasing and installing the technology } & $3,809,524$ & 380,952 & $1,619,048$ & $5,428,571$ & $2,000,000$ \\
\hline \multicolumn{2}{|c|}{ Computer and scanning equipment } & 253,968 & 253,968 & 539,683 & 793,651 & 793,651 \\
\hline \multicolumn{2}{|c|}{ Building } & 178,095 & 75,556 & 134,921 & 313,016 & 210,476 \\
\hline \multicolumn{2}{|l|}{ Staff Costs } & 29,714 & 14,857 & 14,857 & 44,571 & 29,714 \\
\hline \multicolumn{2}{|l|}{ Consumer Goods } & 113,313 & 283,352 & $1,068,019$ & $1,181,331$ & $1,351,371$ \\
\hline \multicolumn{2}{|l|}{ Other Expenses } & 33,533 & 27,944 & 27,944 & 61,477 & 55,888 \\
\hline \multicolumn{2}{|l|}{ Total Annual Costs } & 600,719 & 397,201 & $1,340,185$ & $1,940,903$ & $1,737,386$ \\
\hline \multicolumn{2}{|l|}{ cost per unit } & 96 & 25 & 61 & 158 & 87 \\
\hline
\end{tabular}

Table 4. Summary of the results of the cost-effectiveness analysis of the studied diagnostic parameters of PEM and PET methods

\begin{tabular}{lcccc}
\hline Parameter & Eff $($ Diff $)$ & Costs $\left(\right.$ Diff $\left.^{1}\right) \$$ & Incr $\mathrm{CE}^{2} \$$ & Result \\
\hline Sensitivity & 0.25 & -70.8 & Dominant & PEM dominates \\
Specificity & -0.013 & -70.8 & 5,447 & PEM dominates \\
PPV & 0.15 & -70.8 & Dominant & PEM dominates \\
NPV & 0.072 & -70.8 & Dominant & PEM dominates \\
Accuracy & 0.081 & -70.8 & Dominant & PEM dominates \\
\hline
\end{tabular}

${ }^{1}$ Difference; 2- incremental cost effectiveness ratio (Incremental cost per extra effectiveness) 

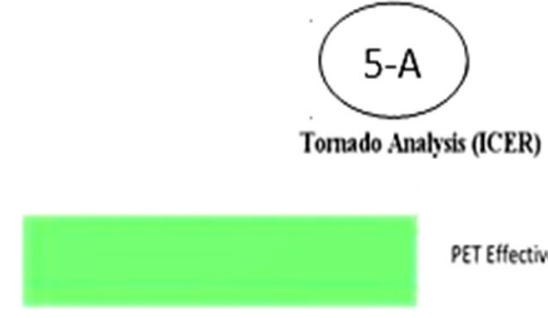

PET Effectiveness

\section{5-B}

Tomado Analysis (ICER)
PET Cost

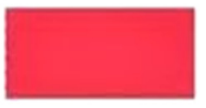

PEM Effectiveness

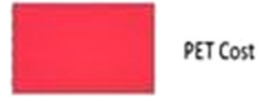

PEM Cost

Ev costese

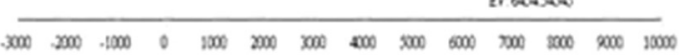

Fig. 5. Tornado diagram illustrating the one-way sensitivity analysis results in terms of sensitivity (5-A) and specificity (5-B)

\$5446. As ICER value is lower than the threshold, PEM diagnostic method is also cost-effective in terms of this index.

\section{Uncertainty analysis}

According to the Tornado diagram in Figure $5 \mathrm{~A}$, the finding showed that ICER had the highest sensitivity to the effectiveness of the PET technology and the lowest sensitivity to the costs of the PEM technology. Also, in Figure $5 \mathrm{~B}$, ICRE had the highest sensitivity to the effectiveness of the PEM and PET technologies, respectively. The lowest sensitivity belonged to the cost of the PEM technology. Therefore, because the ICER values became negative in the cost-effectiveness analysis, it can be concluded that the PEM technology was dominant in both outcomes.

\section{Discussion}

This study is, in fact, the first systematic review of efficacy and cost-effectiveness aimed to evaluate the PEM diagnostic strategy, as compared with PET, in the diagnosis of breast cancer among breast cancer patients in Iran. The results of the meta-analysis showed that the two diagnostic methods are the same in terms of specificity and PPV indices. However, the PEM method is superior to the PET method in the diagnosis of primary breast cancer in terms of sensitivity, NPV, and accuracy. Therefore, the PEM method is preferred to the PET method in terms of efficacy. Comparison of PEM and PET diagnostic methods showed that the difference between the two methods in terms of sensitivity, specificity, PPV, NPV, and accura- cy was $0.25,-0.013,0.15,0.072$, and 0.081 , respectively. As shown, except for the specificity index, the other indices were positive, indicating that the use of PEM for breast cancer patients is better than that of PET. This finding is in line with the results of Yamamoto et al.'s study, which aimed to compare the sensitivity of PEM and PET methods; the authors concluded that the sensitivity of the PEM diagnostic method was higher than that of PET (37). Furthermore, previous studies showed that the PEM diagnostic method, on average, had a sensitivity of $90 \%$ to $91 \%$ and a specificity of $86 \%$ to $93 \%$, as compared with the gold standard method, which is the X-Ray mammography technology. Studies have also shown that the use of a PEM diagnostic device has some potential advantages over mammography, such as the ability to detect and differentiate between malignant and benign tumors, not using $\mathrm{X}$-ray, rapid diagnosis, high precision for obtaining valuable information about the location of the lesion, and identification of suspected lesions in the breast $(19,41$, and 42). In another study by Grankvist et al. in 2012, the sensitivity and specificity of the PET diagnostic method, as compared with the gold standard, were $98 \%$ and $77 \%$ (43).

Concerning the cost, the results showed that when considering the maximum number of scans for both devices, the total annual cost of using PEM and PET was $\$ 1737385.7$ and $\$ 1940903.5$, respectively; and the cost of one-time scan (cost per unit) using PEM and PET devices was $\$ 86.82$ and $\$ 157.63$, respectively. As a result, the costs of PET are higher than PEM; this large amount of difference might be attributed to the difference in the cost 
of the technology purchased on the one hand, and the costs of building and personnel, etc. on the other hand.

Moreover, concerning the cost-effectiveness, the results of the study showed that the PEM device was more effective than PET in terms of sensitivity, accuracy, NPV, and PPV indices, and was less costly. Thus, it is not necessary to calculate ICER because PEM is quite superior and costeffective. Although PET has a slightly higher efficacy than PEM in terms of the specificity index; as the calculated ICER is below the threshold, it is clear that PEM technology is more cost-effective. According to the oneway sensitivity analysis results, the PEM technology was more cost-effective than the PET technology. Therefore, the robustness of the results was confirmed. Also, we did not find any international study comparing PET and PEM in terms of cost-effectiveness to compare our results with theirs. As stated above, in fact, this study is one of the first studies that not only asses the efficacy, but also compares the two methods in terms of cost dimension and costeffectiveness.

One of the limitations of this study was the lack of patients who use this diagnostic method in the country; thus, we did not have clear and fully transparent data on costs. In addition, as the outcomes of the study are not general, to determine the cost-effectiveness of the technology, it is better not to compare them with the cost-effectiveness threshold. However, in order to clarify the topic for policymakers and to justify whether the studied technology is effective or not, we used a threshold that is three times higher than the per capita gross domestic product for nongeneral results.

\section{Conclusion}

The results showed that as compared with PET method, the use of PEM diagnostic method for diagnosis of breast cancer was cost-effective in terms of all the five studied parameters (it was definitely cost-effective for four parameters and was also considered as cost-effective for another index, since ICER was below the threshold). In addition, as compared with the gold standard (x-ray mammography), PEM diagnostic method had acceptable sensitivity and specificity. Thus, because of the potential benefit of using PEM diagnostic method over mammography, it is recommended that this technology should be used along with mammography. Therefore, the use of PEM technology for the diagnosis of primary breast cancer is more cost-effective than PET technology, and because of the wide range of the uses of PET technology in different fields, it is recommended that this method should be used in other areas of priority.

\section{Acknowledgments}

The authors would like to thank Shiraz University of Medical Sciences, Shiraz, Iran, and also Center for Development of Clinical Research of Nemazee Hospital and Dr. Nasrin Shokrpour for editorial assistance.

\section{Funding}

This paper was supported by the Iran National Institute of Health Research, Tehran, Iran. The funding body had no role in the design of the study, collection, analysis, and interpretation of data, and in writing the manuscript.

\section{Conflict of Interests}

The authors declare that they have no competing interests.

\section{References}

1. Cooper J. Occupational Therapy in Oncology and Palliative Care. 2nded, England: John Wiley \& Sons Ltd. 2006:1-8.

2. Situ B. Cancer Facts \& Figures 2015. Atlanta: American Cancer Society. 2015:1-56.

3. Ferlay J, Shin HR, Bray F, Forman D, Mathers C, Parkin DM. Estimates of worldwide burden of cancer in 2008: GLOBOCAN 2008. Int J Cancer. 2010;127(12):2893-917.

4. Klionsky DJ, Abdelmohsen K, Abe A, Abedin MJ, Abeliovich H, Acevedo Arozena A, et al. Guidelines for the use and interpretation of assays for monitoring autophagy (3rd edition). Autophagy. 2016;12(1):1-222.

5. Alzheimer's A. 2016 Alzheimer's disease facts and figures. Alzheimers Dement. 2016;12(4):459-509.

6. Alzheimer's A. 2015 Alzheimer's disease facts and figures. Alzheimers Dement. 2015;11:332-84.

7. Daroudi R, Akbari Sari A, Nahvijou A, Kalaghchi B, Najafi M, Zendehdel K. The Economic Burden of Breast Cancer in Iran. Iran J Public Health. 2015;44(9):1225-33.

8. Abbasalipourkabir R SA, Abdullah R. Cytotoxicity effect of solid lipid nanoparticles on human breast cancer cell lines. Biotechnology. 2011;10(6):528-33.

9. Sadjadi A, Nouraie M, Mohagheghi MA, Mousavi-Jarrahi A, Malekezadeh R, Parkin DM. Cancer occurrence in Iran in 2002, an international perspective. Asian Pac J Cancer Prev. 2005;6(3):359-63.

10. Akbari A, Razzaghi Z, Homaee F, Khayamzadeh M, Movahedi M, Akbari ME. Parity and breastfeeding are preventive measures against breast cancer in Iranian women. Breast cancer (Tokyo, Japan) 2011;18(1):51-5.

11. WHO. Cancer Control, knowledge into action, WHO Guide for Effective programs. 2007. http://www.who.int/cancer/modules/ Prevention\%20Module.pdf

12. Berek JS. Novak's gynecology. 13th ed. Philadelphia: Lippincott williams and wilkins. 2002:1375-82.

13. Abedini M, Allame M, kaviyani A, Motlagh M. Breast disease, clinical examination and diagnostic procedures, particularly physician or health centers. Tehran: Mezrab. 2010.

14. Nover AB, Jagtap S, Anjum W, Yegingil H, Shih WY, Shih WH, et al. Modern breast cancer detection: a technological review. Int $\mathrm{J}$ Biomed Imaging. 2009;2009:902326.

15. Cintolo JA, Tchou J, Pryma DA. Diagnostic and prognostic application of positron emission tomography in breast imaging: emerging uses and the role of PET in monitoring treatment response. Breast Cancer Res Treat. 2013;138(2):331-46.

16. Raylman RR, Majewski S, Smith MF, Proffitt J, Hammond W, Srinivasan A, et al. The positron emission mammography/ tomography breast imaging and biopsy system (PEM/PET): design, construction and phantom-based measurements. Phys Med Biol. 2008;53(3):637-53.

17. Facey K, Bradbury I, Laking G, Payne E. Overview of the clinical effectiveness of positron emission tomography imaging in selected cancers. Health Technol Assess (Winchester, England). 2007;11(44):iii-iv, xi-267.

18. Escalona Lopez S, Blasco Amaro JA, Reza Goyanes M. Effectiveness and safety of positron emission tomography in breast cancer (Structured abstract). Health Technology Assessment Database [Internet]. 2007;(2). Available from: http://onlinelibrary. wiley.com/o/cochrane/clhta/articles/HTA-32011001531/frame.html.

19. Berg WA, Weinberg IN, Narayanan D, Lobrano ME, Ross E, Amodei L, et al. High-resolution fluorodeoxyglucose positron emission tomography with compression ("positron emission mammography") is highly accurate in depicting primary breast cancer. Breast J. 2006;12(4):309-23. 
20. Challa VR Srivastava A, Dhar A, Parshad R, Bal C, Gona RMR, et al. Role of fluorine-18-labeled 2-fluoro-2-deoxy-D-glucose positron emission tomography-computed tomography in the evaluation of axillary lymph node involvement in operable breast cancer in comparison with sentinel lymph node biopsy. Indian J Nucl Med. 2013;28(3):138-43.

21. Benard F, Turcotte E. Imaging in breast cancer: Single-photon computed tomography and positron-emission tomography. Breast Cancer Res. 2005;7(4):153-62.

22. Rosen EL, Turkington TG, Soo MS, Baker JA, Coleman RE. Detection of primary breast carcinoma with a dedicated, large-fieldof-view FDG PET mammography device: Initial experience. Radiology. 2005;234(2):527-34.

23. Kalles V, Zografos GC, Provatopoulou X, Koulocheri D, Gounaris A. The current status of positron emission mammography in breast cancer diagnosis. Breast Cancer (Tokyo, Japan). 2013;20(2):123-30.

24. Tafra L. Positron emission mammography: a new breast imaging device. J Surg Oncol. 2008;97(5):372-3.

25. Kalinyak JE, Schilling K, Berg WA, Narayanan D, Mayberry JP, Rai R, et al. PET-guided breast biopsy. Breast J. 2011;17(2):143-51.

26. Berg WA, Blume JD, Adams AM, Jong RA, Barr RG, Lehrer DE, et al. Reasons women at elevated risk of breast cancer refuse breast MR imaging screening: ACRIN 6666. Radiology. 2010;254(1):7987.

27. Moadel RM. Breast cancer imaging devices. Seminars in nuclear medicine. 2011;41(3):229-41.

28. von Schulthess GK. Cost considerations regarding an integrated CTPET system. Eur Radiol. 2000;10 Suppl 3:S377-80.

29. Samprietro-Colom L, Lach K, Escolar I, Sroka S, Soto M, Cicchetti A, et al. A Handbook and a Toolkit for Hospital-Based Health Technology Assessment. Value Health. 2015;18(7):A556.

30. Health Technology Assessment Committee of the Danish National Board of Health. National strategy for health technology assessment. Copenhagen: National Board of Health. 1996.

31. Bozic KJ, Pierce RG, Herndon JH. Health care technology assessment. Basic principles and clinical applications. J Bone Joint Surg Am. 2004;86-A(6):1305-14.

32. Moher D, Liberati A, Tetzlaff J, Altman DG, Group P. Preferred reporting items for systematic reviews and meta-analyses: the PRISMA statement. PLoS Med. 2009;6(7):e1000097.

33. Whiting PF, Rutjes AW, Westwood ME, Mallett S, Deeks JJ, Reitsma JB, et al. QUADAS-2: a revised tool for the quality assessment of diagnostic accuracy studies. Ann Intern Med. 2011;155(8):529-36

34. Akbari Sari A, Ravaghi H, Mobinizadeh M, Sarvari S. The CostUtility Analysis of PET-Scan in Diagnosis and Treatment of NonSmall Cell Lung Carcinoma in Iran. Iran J Radiol. 2013;10(2):61-7.

35. Assessment IHT. Health Technology Assessment Report (Positron Emission Tomography). 2007. http://ihta.behdasht.gov.ir/uploads/ 354_1720_PET.pdf

36. Yamamoto $\mathrm{Y}$, Tasaki $\mathrm{Y}$, Kuwada $\mathrm{Y}$, Ozawa $\mathrm{Y}$, Inoue T. A preliminary report of breast cancer screening by positron emission mammography. Ann Nucl Med. 2016;30(2):130-7.

37. Yamamoto Y, Ozawa Y, Kubouchi K, Nakamura S, Nakajima Y, Inoue $\mathrm{T}$. Comparative analysis of imaging sensitivity of positron emission mammography and whole-body PET in relation to tumor size. Clin Nucl Med. 2015;40(1):21-5.

38. Kalinyak JE, Berg WA, Schilling K, Madsen KS, Narayanan D, Tartar M. Breast cancer detection using high-resolution breast PET compared to whole-body PET or PET/CT. Eur J Nucl Med Mol I. 2014;41(2):260-75

39. Eo JS, Chun IK, Paeng JC, Kang KW, Lee SM, Han W, et al. Imaging sensitivity of dedicated positron emission mammography in relation to tumor size. Breast (Edinburgh, Scotland). 2012;21(1):6671.

40. Schilling K, Narayanan D, Kalinyak JE, The J, Velasquez MV, Kahn S, et al. Positron emission mammography in breast cancer presurgical planning: comparisons with magnetic resonance imaging. Eur J Nucl Med Mol I. 2011;38(1):23-36.

41. Bergman AM, Thompson CJ, Murthy K, Robar JL, Clancy RL, English MJ. Technique to obtain positron emission mammography images in registration with x-ray mammograms. Med Phys. 1998;25(11):2119-29.

42. Weinberg I, Majewski S, Weisenberger A, Markowitz A, Aloj L, Majewski L, et al. Preliminary results for positron emission mammography: real-time functional breast imaging in a conventional mammography gantry. Eur J Nucl Med. 1996;23(7):804-6.

43. Grankvist J, Fisker R, Iyer V, Frund ET, Simonsen C, Christensen $\mathrm{T}$, et al. MRI and PET/CT of patients with bone metastases from breast carcinoma. Eur J Radiol. 2012;81(1):e13-8. 